\title{
THE LAW OF SUPPLY AND DEMAND
}

\author{
DAVID GALE
}

1. Introduction. The concept of a general economic equilibrium based on balance of supply and demand has from the first played a central role in theoretical economics. In its simplest form the situation can be described roughly in the following terms: In a free market the price of each commodity depends on the extent to which it is demanded by consumers. If at a given set of prices the demand for a good exceeds the available supply then its price rises thus causing the demand to decrease, while if supply exceeds demand the price will drop and demand will thereupon increase. By this mechanism it is supposed that prices will eventually regulate themselves to values at which supply and demand exactly balance, these being the prices at economic equilibrium.

It is only comparatively recently that rigorous investigations have been made as to the conditions under which such a balance is possible. Notable among these are the papers of Wald, especially [5], and most recently some results of Arrow and Debreu [1]. Wald, using methods of analysis, proves an equilibrium theorem under rather special assumptions on the nature of demand functions. We shall here study a model closely resembling that of $[1]$. However, where the latter makes use of some rather sophisticated results of algebraic topology, we shall obtain a simple proof of the existence of an equilibrium using a well-known lemma of elementary combinatorial topology.

In the next section we describe the model to be investigated and state the equilibrium theorem which is proved in Section 3. In Section 4 we apply the principal lemma to a model in which the units are divided into consumers and industries, the consumers sharing profits in industries. In Section 5 a set of conditions which guarantees uniqueness of equilibrium is given. Section 6 deals with an equilibrium theorem for the special case of the Leontief model; the proof is based on linear equations and inequalities thus being independent of the principal theorem. Finally, Sections 7 and 8 are concerned with preference orderings and supply functions originating from such orderings; essentially the first theorem of Arrow and Debreu is obtained.

Received June 8, 1954. 
2. The model. The model to be considered will involve $n$ goods $G_{1}, \ldots, G_{n}$ and $m$ economic units $U_{1}, \ldots, U_{m}$. The set of goods includes all types of labor and services as well as material commodities. The economic units may be thought of as either consumers or as industries, their essential property being that each unit is capable of supplying certain sets of goods while at the same time consuming other sets of goods supplied by the other units. A typical consumer, thus, supplies labor of various types while consuming food, housing, clothes, etc. An industry may supply, for example, consumer goods while consuming various types of raw materials, and so forth.

The amounts of the goods $G_{1}, \ldots, G_{n}$ supplied or consumed by a unit $U$ in a certain fixed time interval may be given by a vector

$$
x=\left(\xi_{1}, \ldots, \xi_{n}\right)
$$

in euclidean $n$-space. The $j^{\text {th }}$ coordinate $\xi_{j}$ represents the amount of the good $G_{j}$ and is positive or negative according as $G_{j}$ is supplied or consumed. Such a vector is called a commodity bundle or an activity of $U$. In general, a unit is able to act in various ways; the set $X$ of all possible commodity bundles $x$ is called the commodity set of the unit.

Definition. A model $M$ consists of a set of goods $G_{1}, \ldots, G_{n}$ and units $U_{1}, \ldots, U_{m}$ with corresponding commodity sets $X_{1}, \ldots, X_{m}$.

Assumption I. The sets $X_{i}$ are closed, bounded and convex and contain the null vector 0 .

The assumption calls for some comment. The condition of boundedness is natural in view of the fixed time interval, and the closedness represents an idealization of a topological character which is not very restrictive when boundedness and convexity are assumed. The condition of convexity is somewhat more restrictive. Together with the fact that the null vector belongs to each $X_{i}$, it means that if $x_{i}$ and $x_{i}{ }^{\prime}$ are two bundles in $X_{i}$ then so also are all bundles of the form $\alpha x_{i}+\beta x_{i}{ }^{\prime}$ where $\alpha, \beta \geqq 0$ and $\alpha+\beta \leqq 1$.

The problem is now to determine how the model $M$ should operate. We must answer the following questions: (a) What goods shall the industries produce? (b) What types of services shall the consumers supply? (c) How shall the goods produced be distributed among the consumers? In our model these three problems are all included in the single question: What bundle $x_{i}$ shall be assigned to the unit $U_{i}$ from the set $X_{i}$ ? Clearly such an assignment of bundles $x_{i}$ to units $U_{i}$ must satisfy the condition that the total amount of each good consumed by all the units must not exceed the total amount supplied. In other words the 
set of bundles $x_{i}$ must satisfy the simple condition $\Sigma x_{i} \geqq 0 .{ }^{1}$ We are thus led to make the following

Definition. A feasible operation of the model $M$ is a set of bundles $x_{i}, i=1, \ldots, m$, where $x_{i} \in X_{i}$ and $\Sigma x_{i} \geqq 0$.

In what follows we shall show how a feasible operation of $M$ can be brought about by the mechanism of prices and free competition. In so doing we shall in addition to answering questions (a)-(c) give an answer to a fourth question: (d) What shall be the relative prices of the goods $G_{j}$ ?

A price vector $p=\left(\pi_{1}, \ldots, \pi_{n}\right) \geq 0$, is an assignment of prices to the goods $G_{j}, \pi_{j}$ being the price of one unit of $G_{j}$. Since only relative prices are significant it is convenient to make the normalizing assumption that $\Sigma \pi_{j}=1$. The set of all price vectors $P$ is thus a regular $(n-1)$-simplex.

We now assume that we have a free competitive economy in which each unit $U_{i}$ may choose a commodity bundle $x_{i}$ which, intuitively speaking, maximizes its satisfaction. This choice will clearly be a function $S_{i}$ of the prices $p$. In the first place, the bundle chosen by $U_{i}$ must be such that the income received from goods supplied is sufficient to pay for the goods consumed. This is the well-known budget inequality which in our notation takes the simple form, $p \cdot S_{i}(p) \geqq 0$, that is, the scalar product of $p$ and $S_{i}(p)$ must be non-negative. Subject to this inequality it is generally assumed that each industry acts so as to maximize profits while each consumer chooses a bundle which maximizes his utility as measured by some appropriate method.

For our present purposes the motivations of the units $U_{i}$ in making their choices is irrelevant, the only requirement being that the functions $S_{i}$ satisfy certain rather weak restrictions. It will, however, be necessary to allow the functions $S_{i}$ to be set-valued rather than ordinary (singlevalued) functions as will be evident from the following discussion. We first give the conditions on the functions $S_{i}$.

Assumptron II. Corresponding to each unit $U_{i}$ there is a function $S_{i}$, called the $i^{\text {th }}$ unit's supply function, defined for each price vector $p$ in $P$ and such that $S_{i}(p)$ is a subset of $X_{i}$ subject to the following restrictions:

(1) $S_{i}(p)$ is a non-empty convex subset of $X_{i}$.

(2) If $x \in S_{i}(p)$ then $x \cdot p \geqq 0$.

(3) $S_{i}$ is continuous in the sense that the graph of $S_{i}$ is a closed subset of $P \times X_{i}$.

1 We adopt the usual conventions, $x>0$ means all coordinates of $x$ are positive, $x \geqq 0$ means all coordinates are non-negative, and $x \geqq 0$ means $x \geqq 0$ but $x \neq 0$. 
Note that the term supply function has been used in a broad sense. The $j^{\text {th }}$ coordinate function $s_{i j}$ of $S_{i}$ represents the amount of $G_{j}$ supplied by $U_{i}$ as a function of prices. If $s_{i j}(p)$ is negative this means that $U_{i}$ supplies a negative amount of $G_{j}$ at prices $p$, meaning, of course, that $U_{i}$ demands a positive amount of $G_{j}$ at these prices. Thus, a negative supply of a good represents a demand for this good.

As to the three conditions, (2) is again the budget inequality. Concerning conditions (1) and (3), for the case where $U$ is an industry, it is reasonable to suppose that $U$ chooses those bundles which will maximize its profit, that is

$$
S(p)=\{x \mid x \in X \text { and } p \cdot x=\text { maximum }\} .
$$

Geometrically, $S(p)$ is the intersection of $X$ with its supporting hyperplane with $p$ as outward normal vector. Since $X$ is bounded, closed and convex, so also is $S(p)$ so that condition (1) is satisfied and it is a simple matter to verify that (2) and (3) are as well. As a simple special case, suppose that $X$ is a line segment of all vectors $\lambda x$ where $x$ is fixed and $0 \leqq \lambda \leqq 1$. Then, according to (4),

$$
S(p)=\left\{\begin{array}{cc}
\{x\} & \text { for } x \cdot p>0 \\
X & \text { for } x \cdot p=0 \\
\{0\} & \text { for } x \cdot p<0
\end{array}\right.
$$

From this we see that it would be too restrictive to require the supply functions to be single valued.

The justification of assumption II when the unit $U$ is a consumer is more involved and will be taken up in Section 7 .

We shall in what follows have occasion to use a strengthened form of assumption II in which the budget inequality is replaced by an equation, thus

$\left(2^{\prime}\right)$ If $x \in S_{i}(p)$ then $x_{i} \cdot p=0$.

This condition means that each of the units spends its entire income on consumption from the other units. Henceforth, when condition $\left(2^{\prime}\right)$ holds for the functions $S_{i}$, we shall say that we have a model without savings.

We can now state the basic equilibrium theorem as follows:

Theorem 1. There exists a price vector $p_{e}$ and a feasible operation $x_{1}, \ldots, x_{m}$ of $M$ such that $x_{i} \in S_{i}\left(p_{e}\right)$ for $i=1, \ldots, m$.

We shall henceforth refer to $p_{e}$ as an equilibrium price vector and the bundles $x_{i}$ as equilibrium bundles.

In concrete terms, this theorem states that if prices are appropriately set then it is possible for each unit to be assigned a commodity bundle of its choice among those bundles which it can afford. 
Note that our model includes as a special case the situation of pure exchange. This is the case of a group of individuals each initially possessing various amounts of the goods $G_{j}$. If we assume some or all of the participants can increase their satisfaction by exchanging some of their holdings, the equilibrium theorem shows how such an exchange can be brought about by the mechanism of prices. A special case of this situation was treated by Wald in [5].

Theorem 1 is a simple corollary of the following abstract lemma which is the central mathematical result of this paper.

Principal lemma. Let $S$ be a bounded continuous set-valued function from the unit $(n-1)$-simplex $P$ into $R_{n}$ such that

(a) $S(p)$ is non-empty and convex for all $p \in P$,

(b) if $x \in S(p)$ then $x \cdot p \geqq 0$.

Then there exists $p \in P$ and $x \in S(p)$ such that $x \geqq 0$.

This lemma will be proved in the next section. We shall now show how the lemma implies theorem 1.

For the model $M$ with commodity sets $X_{i}$ and supply functions $S_{i}$, we define the aggregate commodity set $X_{M}$ by

$$
X_{M}=\sum_{i=1}^{m} X_{i}=\left\{x \mid x=\sum_{i=1}^{m} x_{i}, x_{i} \in X_{i}\right\}
$$

and the aggregate supply function by

$$
S_{M}(p)=\sum_{i=1}^{m} S_{i}(p)=\left\{x \mid x=\sum_{i=1}^{m} x_{i}, x_{i} \in S_{i}(p)\right\} .
$$

Since the functions $S_{i}$ satisfy assumption II one verifies at once that $S_{M}$ satisfies the hypothesis of the principal lemma. In view of the lemma, therefore there exist vectors $p \in P$ and $x \in S_{M}(p)$ such that $x \geqq 0$. Now this $x$ is of the form $x=\sum x_{i}$ where $x_{i} \in S_{i}(p)$ and hence the bundles $x_{i}$ give the desired feasible operation of the model.

We conclude this section with the following observation. Theorem 1 states only that at equilibrium supply is at least equal to demand, not that the two exactly balance. One cannot in general expect perfect balance. For example, a given process of production might involve supplying certain by-products of which the total output is not required by consumers at equilibrium. In this case a certain amount of over-production would be inevitable. Now suppose that $p_{e}=\left(\pi_{1}, \ldots, \pi_{n}\right)$ is an equilibrium price vector and $x_{i}$ are corresponding equilibrium bundles. Let $x=\Sigma x_{i}=\left(\xi_{1}, \ldots, \xi_{n}\right)$. We say that $G_{k}$ is over-produced at equilibrium if $\xi_{k}>0$. Since there is in this case a surplus of $G_{j}$ one would expect its 
price to drop to zero. This is indeed true for the important special case of a model without savings. We then have the following:

REMARK. If the supply functions $S_{i}$ satisfy the sharpened condition $\left(2^{\prime}\right)$, then if $G_{k}$ is over-produced at equilibrium its price is zero.

To see this, note that since $x_{i} \cdot p=0$ for all $i$, we have $\left(\sum x_{i}\right) \cdot p=x \cdot p=0$ or

$$
\sum_{j=1}^{n} \xi_{j} \pi_{j}=0
$$

Since all $\xi_{j}$ and $\pi_{j}$ are non-negative it follows that if $\xi_{k}>0$ then $\pi_{k}=0$. We thus have the fact that a good which is over-produced at equilibrium becomes a free good, a result which has been well known to economists for some time.

3. Proof of the principal lemma. The tool needed to prove the principal lemma is a well-known result of Knaster, Kuratowski and Mazurkiewicz [4] on coverings of simplexes (lemma 1 below). It was used by these authors to prove the Brouwer fixed point theorem and still represents the most direct way of obtaining that classical result.

Consider the $(n-1)$-simplex $P$ in $R_{n}$ consisting of all points $p=$ $\left(\pi_{1}, \ldots, \pi_{n}\right)$ such that $p \geqq 0$ and $\sum \pi_{j}=1$. If $J$ is a subset of the indices $1, \ldots, n$, a face $F_{J}$ of $P$ is defined by

$$
\boldsymbol{F}_{\boldsymbol{J}}=\left\{p \mid p \in P \text {, and } \pi_{j}=0 \text { for } j \notin J\right\} .
$$

Lemma 1. Let $C_{1}, \ldots, C_{n}$ be a family of closed subsets of $P$ with the property that if $F_{J}$ is any face of $P$ then $F_{J} \subset \cup_{j \in J} C_{j}$. Then the sets $C_{j}$ have a non-empty intersection.

A simple illustration of the lemma is the case of a triangle covered by three closed sets such that the set containing a given vertex does not intersect the opposite side. For a self-contained proof of the lemma see [2, pp. 145-149].

We can now easily prove the principal lemma for the special case where the function $S$ is single valued and hence continuous in the ordinary sense. For this case let $s_{j}$ be the $j^{\text {th }}$ coordinate function of $S$.

Define $C_{j}=\left\{p \mid s_{j}(p) \geqq 0\right\}$. Since $s_{j}$ is continuous, $C_{j}$ is closed.

Let $K$ be a subset of the indices $1, \ldots, n$, say, $K=\{j \mid j \leqq k\}$, and let $F_{K}$ be the corresponding face of $P$. If $p \in F_{K}$ then we may write $p=$ $\left(\pi_{1}, \ldots, \pi_{k}, 0, \ldots, 0\right)$. Now from condition $(\mathrm{b}), S(p) \cdot p \geqq 0$, or

$$
\sum_{j=1}^{n} s_{j}(p) \pi_{j}=\sum_{j=1}^{k} s_{j}(p) \pi_{j} \geqq 0 .
$$


Since $\pi_{j} \geqq 0$ for all $j$, it follows that $s_{j}(p) \geqq 0$ for at least one $j \in K$. Thus $p \in \mathrm{U}_{j \in K} C_{j}$ or $F_{K} \subset \mathrm{U}_{j \in K} C_{j}$, so the hypothesis of lemma 1 is satisfied. It follows that there exists $p \in \bigcap_{j=1}^{n} C_{j}$, and for this vector $S(p) \geqq 0$ as was to be shown.

The proof for the general case is a technical matter of approximating the function $S$ by single-valued functions in a manner similar to that used by Kakutani in [3].

If $p \in P$ and $x \in R_{n}$, we shall denote by $|p|$ and $|x|$ the euclidean norms of these vectors, that is, $|x|=(x \cdot x)^{\frac{1}{2}}$. For any real number $\alpha$, the symbol $[\alpha]$ denotes the vector all of whose coordinates are equal to $\alpha$.

Since the function $S$ is bounded there exists an upper bound $\mu$ such that $|x| \leqq \mu$ for all $x \in S(p)$ and all $p \in P$.

We now proceed with the approximation process. For each positive integer $k$ let $\Lambda_{k}$ be a simplicial subdivision of $P$. Let $\delta_{k}$ denote the maximum diameter of the subsimplexes of $\Lambda_{k}$, and choose $\Lambda_{k}$ so that $\delta_{k} \leqq$ $1 /(\mu k)$.

We now define a sequence of single-valued functions $S^{k}$ on $P$ as follows: If $p$ is a vertex of $\Lambda_{k}$ choose $S^{k}(p)$ to be a point in the set $S(p)+[1 / k]$. Extend $S^{k}$ to the rest of $P$ linearly in every simplex of $\Lambda_{k}$. Then $S^{k}$ is single valued and continuous. We shall now show that condition (b) of the principal lemma is also satisfied, that is, $S^{k}(p) \cdot p \geqq 0$ for all $p \in P$.

For any $p \in P$ we may write $p=\Sigma \lambda_{j} p_{j}$ where $\lambda_{j} \geqq 0, \Sigma \lambda_{j}=1$, and $p_{j}, j=1, \ldots, n$, are the vertices of a simplex of the subdivision $\Lambda_{k}$, so $\left|p_{i}-p_{j}\right| \leqq \delta_{k}$. Let $x_{j}=S^{k}\left(p_{j}\right)$ and let $x=S^{k}(p)=\Sigma \lambda_{j} x_{j}$. Then $\left|p-p_{j}\right| \leqq$ $\leqq \delta_{k} \leqq 1 /(\mu k)$ and $\left|x_{j}\right| \leqq \mu$, so from the Schwarz inequality

$$
p_{j} \cdot x_{j}-p \cdot x_{j}=\left(p_{j}-p\right) \cdot x_{j} \leqq 1 / k .
$$

On the other hand, $p_{j} \cdot x_{j} \geqq 1 / k$ from the definition of $S^{k}$, hence it follows that $p \cdot x_{j} \geqq 0$. Since $x=\Sigma \lambda_{j} x_{j}$ and $\lambda_{j} \geqq 0$, we conclude that $p \cdot x \geqq 0$.

It now follows from the proof of the principal lemma for the singlevalued case that for each $k$ there exists a vector $p^{k}$ such that $S^{k}\left(p^{k}\right) \geqq 0$. Let $p^{k}=\sum_{j=1}^{n} \lambda_{j}^{k} p^{k}{ }_{j}$, where $p^{k}{ }_{j}$ are vertices of a simplex of $\Lambda_{k}$ containing $p^{k}$. Let $x^{k}=S^{k}\left(p^{k}\right)$ and $x^{k}{ }_{j}=S^{k}\left(p^{k}{ }_{j}\right)$. Since $P$ is compact and $S$ is bounded we may by choosing convergent subsequences assume that the sequences $p^{k}, x^{k}, p^{k}{ }_{j}$, and $x^{k}{ }_{j}$ converge. Let $p^{k} \rightarrow p$. Then also $p^{k}{ }_{j} \rightarrow p$, since $\delta_{k} \rightarrow 0$. Let $x^{k}{ }_{j} \rightarrow x_{j}$. Now $x^{k}{ }_{j}=y^{k}+[1 / k]$ where $y^{k}{ }_{j} \in S\left(p^{k}{ }_{j}\right)$, hence $y^{k_{j}} \rightarrow x_{j}$. Since $S$ is continuous, it follows that $x_{j} \in S(p)$. Finally, $x^{k} \rightarrow x$. Since $x^{k} \geqq 0$, so also is $x$, and since $x^{k}$ lies in the convex hull of the points $x^{k}$, we have in the limit that $x$ lies in the convex hull of the points $x_{j}$. Thus, since $S(p)$ is convex, we conclude that $x \in S(p)$ and the proof is complete. 
4. Industries and profits. The model of [1] differs from the one treated here in that there the economic units are explicitly divided into the two classes, industries and consumers ("production" and "consumption" units). The industries act to maximize profits, the consumers to maximize utility. To close the model, profits from industries are redistributed to consumers who are thought of as holding stock in the industries. We shall see that an equilibrium theorem for this more general model follows readily from our principal lemma.

Let the economic units $U_{1}, \ldots, U_{m}$ be divided into two classes, industries $I_{1}, \ldots, I_{s}$ and consumers $C_{1}, \ldots, C_{t}$, where $s+t=m$. Further, let each consumer $C_{k}$ be entitled to a share $\alpha_{i k}$ of the profits from the industry $I_{i}$ where

$$
\sum_{k=1}^{t} \alpha_{i k}=1 \text { for all } i
$$

meaning that the profits from each industry are to be completely distributed among the consumers. Then the income the consumer $C_{k}$ obtains from the industries is $\left(\sum_{i=1}^{s} \alpha_{i k} x_{i}\right) \cdot p$.

Let $S_{i}$ be the supply function of $I_{i}$ and $T_{k}$ the supply function of $C_{k}$ and let $X_{i}$ and $Y_{k}$ be the corresponding commodity sets. We again assume conditions (1) and (3) of assumption II. Condition (2) must, however, be changed to take account of the additional income to consumers. The new assumption is:

(2a) For industries, if $x_{i} \in S_{i}(p)$ then $x_{i} \cdot p \geqq 0$.

(2b) For consumers, if $y_{k} \in T_{k}(p)$ then

$$
y_{k} \cdot p+\left(\sum_{i=1}^{s} \alpha_{i k} x_{i}\right) \cdot p \geqq 0 .
$$

The equilibrium theorem for this model then takes the following form.

THEOREM 2. There exists a price vector $p_{e}$ and a feasible operation $x_{1}, \ldots, x_{s}, y_{1}, \ldots, y_{t}$ of the model such that $x_{i} \in S_{i}\left(p_{e}\right)$ for $i=1, \ldots, s$ and $y_{k} \in T_{k}\left(p_{e}\right)$ for $k=1, \ldots, t$.

Proof. Consider the aggregate supply function

$$
\begin{aligned}
S(p) & =\sum_{i=1}^{s} S_{i}(p)+\sum_{k=1}^{t} T_{k}(p) \\
& =\left\{z \mid z=\sum_{i=1}^{s} x_{i}+\sum_{k=1}^{t} y_{k}, x_{i} \in S_{i}(p), y_{k} \in T_{k}(p)\right\} .
\end{aligned}
$$

We must show that $S$ satisfies the hypothesis of the principal lemma, in particular condition (b) (the other conditions follow at once). To see 
this, sum inequalities (6) over all indices $k$ and use (5) to obtain

$$
\left(\Sigma y_{k}+\Sigma x_{i}\right) \cdot p \geqq 0
$$

which is the desired relation.

The principal lemma now implies theorem 2 by the same argument as was used in proving theorem 1.

REMARK 1. Note that condition (2a) was not used in the proof of theorem 2 which is therefore valid even if industries are allowed to operate at a loss, provided this loss is distributed among the consumers holding shares.

REMARK 2. In the important special case of a model with no savings by consumers, inequalities $(2 b)$ become equations and in order for these equations to hold the quantity $\left(\sum_{i=1}^{s} \alpha_{i k} x_{i}\right) \cdot p$ must have the same value for all bundles $x_{i} \in S_{i}(p)$. This will be true, for example, if each industry acts to maximize profits so that $S_{i}(p)=\left\{x \mid x \in X_{i}\right.$ and $x \cdot p=$ maximum $\}$.

5. A remark on uniqueness of equilibrium. It is naturally of interest to determine conditions under which the equilibrium prices and bundles will be uniquely determined. If one makes the assumption that an increase in the relative price of a good causes a decrease in the net demand for that good (thus an increase in the net supply) then one can give a very simple uniqueness proof.

Theorem 3. Hypotheses:

(a) The functions $S_{i}$ are single valued.

(b) Condition (2') holds.

(c) If prices $\pi_{j_{1}}, \ldots, \pi_{j_{r}}$ increase while all others decrease or remain fixed, then the net supply of at least one good $G_{j_{1}}, \ldots, G_{j_{r}}$ will increase.

Conclusion: The equilibrium is unique.

Proof: Let $p$ be an equilibrium price vector and let $x_{i}=S_{i}(p)$. Let $\sum x_{i}=x=\left(\xi_{1}, \ldots, \xi_{n}\right)$. Suppose now $p^{\prime} \neq p$, and let $y_{i}=S_{i}\left(p^{\prime}\right)$ and $\Sigma y_{i}=y=\left(\eta_{i}, \ldots, \eta_{n}\right)$. From (c) it follows that for at least one index $j$, say, $j=1$, we have $\pi_{1}{ }^{\prime}>\pi_{1}$ and $\eta_{1}>\xi_{1}$. From (b) we get,

$$
\sum \eta_{j} \pi_{j}{ }^{\prime}=0 \text {. }
$$

Since the $x_{i}$ yield a feasible operation, $\xi_{1} \geqq 0$, hence $\eta_{1}>0$, and since $\pi_{1}{ }^{\prime}>0,(7)$ implies that for at least one index $j, \eta_{j}<0$. This means that the bundles $y_{i}$ do not give a feasible operation, hence the equilibrium is unique. 
The hypotheses of theorem 3 are rather restrictive and may fail to hold in practical cases. The problem of finding reasonable conditions on the supply functions which will insure uniqueness would seem to offer a significant field for further study.

6. An example. As a concrete illustration of the equilibrium concept we here apply it to the special case of the so-called Leontief model. For this special case we give a self-contained proof of the existence of equilibrium. In this model there are $n+1$ goods, $G_{0}, G_{1}, \ldots, G_{n}$, where $G_{0}$ is labor and $G_{j}, j>0$, are various material goods. The economic units consist of $n$ industries, $I_{1}, \ldots, I_{n}$, and one consumption unit $C_{0}$.

We make the following assumptions:

(i) Each industry $I_{i}$ is able to supply (produce) only the good $G_{i}$. The only good which $C_{0}$ can supply is labor, $G_{0}$.

(ii) Labor is required as an input to each industry $I_{i}$. $C_{0}$ can supply at most the amount $\alpha_{0}$ of $G_{0}$.

(iii) The commodity sets $X_{i}, i=1, \ldots, n$, consist of line segments. If $\alpha_{i j}$ is the amount of $G_{j}$ supplied by $I_{i}$ when consuming one unit of labor, then $X_{i}$ consists of all bundles of the form $x_{i}=$ $\lambda\left(-1, \alpha_{i 1}, \ldots, \alpha_{i n}\right)$, where $0 \leqq \lambda \leqq \alpha_{0}$. Thus each industry can produce at any level, $\lambda$, provided that it does not consume more than the total available supply of labor. Note that from condition (i) $\alpha_{i j} \leqq 0$ for $i \neq j$.

(iv) The industries $I_{i}$ are able to supply simultaneously a positive amount of each good $G_{j}, j>0$. In other words, there exist numbers $\mu_{i}>0$, such that $\sum_{i=1}^{n} \mu_{i} \alpha_{i j}>0$ for all $j>0$.

Condition (i) states that there is no joint production in the model. Condition (ii) is self-explanatory. Condition (iii) says that there is essentially only one process for producing each good; and condition (iv) is the requirement that it be possible to make all goods $G_{j}$ available for consumption. In addition we assume

(v) Each industry acts so as to maximize profits. In other words

$$
S_{i}(p)=\left\{x_{i} \mid x_{i} \in X_{i} \text { and } x_{i} \cdot p=\operatorname{maximum}\right\} \text { for } i>0 .
$$

The commodity set $X_{0}$ of $C_{0}$ can be an arbitrary compact convex set subject only to conditions (i) and (ii). That is, $x_{0}=\left(\xi_{0}, \xi_{1}, \ldots, \xi_{n}\right) \in X_{0}$ implies $\xi_{0} \leqq \alpha_{0}$ and $\xi_{j} \leqq 0$ for all $j>0$. For the theorem that follows the supply function $S_{0}$ can be quite arbitrary subject only to the budget inequality.

Subject to these conditions we shall show that there exists an equilibrium in the sense of theorem 1 satisfying the additional conditions: 
(A) All prices $\pi_{j}$ are positive.

(B) Profits are zero for each industry $I_{i}$. We state this result in the form of a theorem as follows.

Theorem 4. If $M$ is a model satisfying conditions (i)-(v), then there exist a unique equilibrium price vector $p_{0}$ and bundles $y_{0}, x_{1}, \ldots, x_{n}$ such that $p_{0}>0$ and $p_{0} \cdot x_{i}=0$ for $i=1, \ldots, n$.

We shall give a self-contained proof of this theorem which is independent of theorem 1 and is elementary, that is, does not require results from topology. For this purpose we shall need some preliminary lemmas.

LEMMA 2. Let $A$ be an $n \times n$ matrix with coefficients $\alpha_{i j}$ sueh that $\alpha_{i j} \leqq 0$ for $i \neq j$ and suppose there exists a vector $x>0$ such that $x A>0$. Then $y A \geqq 0$ implies $y \geqq 0$.

Proof. Let $x=\left(\xi_{1}, \ldots, \xi_{n}\right), y=\left(\eta_{1}, \ldots, \eta_{n}\right)$ and let $\theta=\min _{i}\left(\eta_{i} / \xi_{i}\right)$, say, $\theta=\eta_{1} / \xi_{1}$. Then $\xi_{i} \theta \leqq \eta_{i}$ for all $i$, so $\alpha_{i 1} \eta_{i} \leqq \alpha_{i 1} \xi_{i} \theta$ for $i \neq 1$ and $\alpha_{11} \eta_{1}=$ $\alpha_{11} \xi_{1} \theta$. Therefore $0 \leqq \sum_{i} \alpha_{i 1} \eta_{i} \leqq \theta \sum_{i} \alpha_{i 1} \xi_{i}$. Since $\Sigma \alpha_{i 1} \xi_{i}$ is positive by hypothesis, it follows that $\theta \geqq 0$ and hence $\eta_{i} \geqq 0$ for all $i$.

LeMmA 3. If $A$ is an $n \times n$ matrix such that $y A \geqq 0$ implies $y \geqq 0$, then $A$ is non-singular and $p A^{\prime}>0$ implies $p>0$.

Proof. By hypothesis, $y A=0$ implies $y \geqq 0$, but also $-y \geqq 0$ since $-y A=0$, and so $y=0$. This shows that $A$ is non-singular. Let now $y_{j}$ be such that $y_{j} A=(0, \ldots, 0,1,0, \ldots, 0)$ with 1 at the $j^{\text {th }}$ place, $j=$ $=1,2, \ldots, n$. Then $y_{j} \geqq 0$ by hypothesis and hence $y_{j} \geq 0$, since $y_{j} A \neq 0$ implies $y_{j} \neq 0$. Putting $p=\left(\pi_{1}, \ldots, \pi_{n}\right)$ we obtain from $p A^{\prime}>0$ by multiplication by $y_{j}{ }^{\prime}$ from the right, $\pi_{j}=p A^{\prime} y_{j}{ }^{\prime}>0$ for $j=1,2, \ldots, n$.

Proof of THEorem 4. Let $A$ be the matrix whose entries are $\alpha_{i j}$, $i, j=1, \ldots, n$, and let $a_{i}=\left(\alpha_{i 1}, \ldots, \alpha_{i n}\right)$. From conditions (iii) and (iv), $A$ satisfies the hypothesis of lemma 2 . Since prices are only determined up to a positive factor we may make the normalizing assumption that the price of labor is unity, that is, $\pi_{0}=1$. From lemma $3, A$ is non-singular and there exists one and only one non-negative (price) vector $p=\left(\pi_{1}, \ldots, \pi_{n}\right)$ such that $p \cdot a_{i}=\sum_{j} \pi_{j} \alpha_{i j}=1$ for all $i$. This is just the statement that the profit to each industry $I_{i}$ is zero. Since $\alpha_{i j} \leqq 0$ for $i \neq j$, it follows that $\pi_{i}>0$, hence $p>0$. Now let $y_{0}=\left(\eta_{0},-\eta_{1}, \ldots,-\eta_{n}\right) \in S_{0}\left(p_{0}\right)$ where $p_{0}=\left(1, \pi_{1}, \ldots, \pi_{n}\right)$ and $\eta_{0} \leqq \alpha_{0}$, and let $y=\left(\eta_{1}, \ldots, \eta_{n}\right)$. Again by the non-singularity of $A$ there exist numbers $\lambda_{i}$ such that $\Sigma \lambda_{i} a_{i}=y$, and since $y \geqq 0$ it follows from lemma 2 that $\lambda_{i} \geqq 0$ for all $i$. Since $p \cdot a_{i}=1$ for all $i$, we have $p \cdot \Sigma \lambda_{i} a_{i}=\Sigma \lambda_{i}=p \cdot y$, but $p \cdot y \leqq \eta_{0}$ from 
the budget inequality for $C_{0}$. Now let $x_{i}=\lambda_{i}\left(-1, \alpha_{i 1}, \ldots, \alpha_{i n}\right)$. Since all profits are zero, it follows that $x_{i}$ maximizes profits to $I_{i}$, hence $x_{i} \in S_{i}\left(p_{0}\right)$. Finally we see that $y_{0}+\sum x_{i} \geqq 0$, since $\eta_{0}-\sum \lambda_{i} \geqq 0$, and $-\eta_{j}+\sum_{i} \lambda_{i} \alpha_{i j}=0$ for all $j>0$. We have, therefore, a feasible operation of $M$, and the proof is complete.

Observe that in this example the equilibrium prices $p$ are quite independent of the consumer's preference, being a function only of the nature of the industries $I_{i}$.

7. Supply functions and preference orderings. We now return to the problem mentioned in Section 2, namely that of justifying the conditions of assumption II for the supply functions of consumers. Since the validity of the principal lemma hinges on these conditions some further discussion of them seems in order. We shall show here that they can be derived from simple assumptions concerning the preferences of the consumers.

We assume again that the commodity space $X$ of the unit $U$ is a compact convex subset of $R_{n}$ and assume that $U$ orders the bundles of $X$ by a simple ordering relation $\succsim$. The formula $x \succsim y$ means that the bundle $x$ is at least as satisfactory to $U$ as the bundle $y$. Since $\succsim$ is a simple ordering it satisfies:

(a) $x \succsim y$ and $y \succsim z$ implies $x \succsim z$.

(b) For any $x, y \in X$, either $x \succsim y$ or $y \succsim x$.

If both $x \succsim y$ and $y \gtrsim x$ we write $x \sim y$, while if $x \succsim y$ and $x \sim y$ we write $x \succ y$ (in words, $x$ is preferred to $y$ ).

Definitions. The ordering $\succsim$ is called continuous if $x>y$ implies there exist neighborhoods $U$ of $x$ and $V$ of $y$ such that

$$
x^{\prime}>y^{\prime} \text { for all } x^{\prime} \in U \text { and all } y^{\prime} \in V .
$$

(It is easy to show that this condition is equivalent to an apparently weaker one, namely the existence of neighborhoods $U^{\prime}$ of $x$ and $V^{\prime}$ of $y$ such that $x^{\prime} \succ y$ for all $x^{\prime} \in U^{\prime}$ and $x>y^{\prime}$ for all $y^{\prime} \in V^{\prime}$.)

The ordering $\succsim$ is called convex (strictly convex) if $x \sim y$ and $0<\lambda<1$ implies $\lambda x+(1-\lambda) y \succsim x,(\succ x)$.

Let $S^{+}(x)=\left\{x^{\prime} \mid x^{\prime} \succsim x\right\}, S^{-}(x)=\left\{x^{\prime} \mid x \succsim x^{\prime}\right\}$.

The following property follows at once from the definitions.

Lemma 4. The ordering $\succsim i s$ continuous if and only if $S^{+}(x)$ and $S^{-}(x)$ are closed for all $x \in X$.

Not quite so obvious is the following: 
LemMa 5. A continuous ordering $\succsim$ is convex if and only if $S^{+}(x)$ is convex for all $x \in X$.

Proof. The sufficiency of the condition is obvious. Conversely, suppose $y, z \in S^{+}(x)$, thus $y, z \succsim x$. Let $[y, z]$ denote the segment from $y$ to $z$ and suppose there exists $w \in[y, z]$ such that $x>w$. Since $X$ is convex $[y, z] \in X$. Let $\sigma^{+}=S^{+}(x) \cap[w, y], \sigma^{-}=S^{-}(x) \cap[w, y]$. Since $[w, y]$ is connected and $\sigma^{+}$and $\sigma^{-}$are closed and non-empty, there is a point $y^{\prime} \in \sigma^{+} \cap \sigma^{-}$, so $y^{\prime} \sim x$. Similarly, there is a point $z^{\prime} \in[w, z]$ such that $z^{\prime} \sim x$. But $w \in\left[y^{\prime}, z^{\prime}\right]$ and, since $\succsim$ is convex, $w \gtrsim y^{\prime} \sim x$, giving a contradiction.

If $C$ is a subset of $X$, a point $c \in C$ is called maximal if $c \gtrsim x$ for all $x \in C$.

Lemma 6. If $C$ is a closed subset of $X$ and $\succsim$ is continuous then $C$ contains a maximal element.

Proof. For each $x \in C$, let $C^{+}(x)=S^{+}(x) \cap C$. The sets $C^{+}(x)$ are closed and nested by inclusion, hence, by the compactness of $C$ there exists $c \in \mathrm{\cap}_{x \in C} C^{+}(x)$. This is the desired maximal element.

Definition, Let $p$ be a price vector and let $\gtrsim$ be the preference ordering of the unit $U$ assumed to be continuous. Let $C(p)=\{x \mid x \in X, x \cdot p \geqq 0\}$, thus $\mathrm{C}(p)$ is the set of all bundles satisfying the budget inequality at prices $p$. The supply function $S$ of the unit $U$ is then defined by

$$
S(p)=\{x \mid x \text { is maximal in } C(p)\} .
$$

The following two theorems establish the connection between preference orderings and supply functions.

Theorem 5. If $\succsim$ is convex then $S(p)$ is convex for all $p \in P$. If $\succsim$ is strictly convex then $S$ is single valued.

Proof. Suppose $x, x^{\prime} \in S(p)$. Then $x, x^{\prime} \in C(p)$ and $\left(\lambda x+(1-\lambda) x^{\prime}\right) \cdot p=$ $\lambda x \cdot p+(1-\lambda) x^{\prime} \cdot p \geqq 0$ so $\lambda x+(1-\lambda) x^{\prime} \in C(p)$. If $\succsim$ is convex then $\lambda x+(1-\lambda) x^{\prime} \succsim x$ and since $x$ is maximal in $C(p)$ we must have $\lambda x+(1-\lambda) x^{\prime} \sim x$ so $\lambda x+(1-\lambda) x^{\prime} \in S(p)$. If $\succsim$ is strictly convex then $x=x^{\prime}$; for otherwise we would have $\frac{1}{2}\left(x+x^{\prime}\right)>x$ contradicting the maximality of $x$.

In order to show that $S$ is continuous we are forced to make an additional and somewhat unnatural assumption on the set $X$.

Assumption III. The set $X$ contains a positive vector, that is, there exists $x_{0} \in X$ such that $x_{0}>0$.

This amounts to assuming that $U$ can supply a positive (though arbitrarily small) amount of each good $G_{j}$. This same assumption was made in [1] in proving the first equilibrium theorem of that paper. 
THEOREM 6. If $\succsim$ is continuous and $X$ satisfies III, then $S$ is continuous.

Proof. We must show: if $p^{k} \rightarrow p$ and $x^{k} \rightarrow x$ and $x^{k} \in S\left(p^{k}\right)$ then $x \in \boldsymbol{S}(p)$.

First, since $x^{k} \cdot p^{k} \geqq 0$ for all $k$, it follows from the continuity of the scalar product that $x \cdot p \geqq 0$, so $x \in C(p)$. If $x \notin S(p)$ then there exists $y \in C(p)$ such that $y \succ x$. By continuity of $\succsim$ we can find a neighborhood $N(y)$ such that $y^{\prime} \in N(y)$ implies $y^{\prime} \succ x$. In fact, by choosing $N(y)$ sufficiently small and taking a subsequence of the $x^{k}$ if necessary, we may assume $y^{\prime}>x^{k}$ for all $k$. This means that $N(y) \cap C\left(p^{k}\right)$ is empty for all $k$, for if $y^{\prime} \in N(y) \cap C\left(p^{k}\right)$ then $x^{k} \succsim y^{\prime}$ since $x^{k}$ is maximal in $C\left(p^{k}\right)$. It follows that $y \cdot p^{k}<0$ and $y \cdot p=0$.

Now for the bundle $x_{0}$ of assumption III, we have $x_{0} \cdot p^{k}>0$ since $x_{0}>0$. Define $y^{k}=\left(\left(x_{0} \cdot p^{k}\right) y-\left(y \cdot p^{k}\right) x_{0}\right) / \lambda^{k}$ where $\lambda^{k}=\left(x_{0}-y\right) \cdot p^{k}>0$. Since $X$ is convex, $y^{k} \in X$. Also $y^{k} \cdot p^{k}=0$ so $y^{k} \in C\left(p^{k}\right)$. As $p^{k} \rightarrow p$, $y \cdot p^{k} \rightarrow y \cdot p=0$, hence $y^{k} \rightarrow y$. Thus for $k$ sufficiently large $y^{k} \in N(y)$. This, however, contradicts the fact that $N(y) \cap C\left(p^{k}\right)$ is empty, and the theorem is proved.

To see why some condition such as III is necessary in order to prove continuity of $S$, suppose $G_{1}$ and $G_{2}$ are goods which $U$ is not able to supply. Let

$$
p^{k}=\left(\pi_{1}^{k},\left(1-\pi_{1}^{k}\right), 0, \ldots, 0\right)
$$

where $\pi^{k}{ }_{1}>0, \pi^{k}{ }_{1} \rightarrow 0$, so $p^{k} \rightarrow p=(0,1,0, \ldots, 0)$. Then clearly $S\left(p^{k}\right)$ is constant since $C\left(p^{k}\right)$ is independent of $k$ and if $x^{k}=\left(\xi^{k}{ }_{1}, \ldots, \xi_{n}^{k}\right) \in S\left(p^{k}\right)$ then $\xi^{k}{ }_{1}=0$, since $\xi_{1}^{k}<0$ would mean $x^{k} \cdot p^{k}<0$. On the other hand, if $G_{1}$ is a good which is desired by $U$ then $y=\left(\eta_{1}, \ldots, \eta_{n}\right) \in S(p)$ implies $\eta_{1}<0$. Thus, if we take a convergent subsequence of the $x^{k}$ the limit point $x=\left(\xi_{1}, \ldots, \xi_{n}\right)$ will have $\xi_{1}=0$, so $x \notin S(p)$ and therefore $S$ is not continuous.

8. Equilibrium and optimality. We consider a model with commodity sets $X_{1}, \ldots, X_{m}$, each of which is ordered by a preference ordering $\succsim . A$ feasible operation $x_{1}, \ldots, x_{m}$ of $M$ is called optimal if there is no other feasible operation $y_{1}, \ldots, y_{m}$ of $M$ such that $y_{i} \succsim x_{i}$ in the ordering of $U_{i}$ for every $i=1, \ldots, m$, and $y_{i}>x_{i}$ for at least one unit $U_{i}$.

We are going to prove that, under certain conditions, any equilibrium operation of $M$ is optimal. This will in fact be the case when there is no saving at equilibrium. 
We assume that the ordering of each $X_{i}$ is continuous and convex, and that the supply function $S_{i}$ of each $U_{i}$ is defined as in the preceding section. Thus, if

then

$$
C_{i}(p)=\left\{x_{i} \mid x_{i} \in X_{i}, x_{i} \cdot p \geqq 0\right\}
$$

$$
S_{i}(p)=\left\{x_{i} \mid x_{i} \text { is maximal in } C_{i}(p)\right\} \text {. }
$$

For such a model $M$ the following theorem holds.

Theorem 7. Let $p$ be an equilibrium price vector with the property that if $x_{i} \in S_{i}(p)$ then $x_{i}$ satisfies the budget equality $x_{i} \cdot p=0$. Then any equilibrium operation $x_{1}, \ldots, x_{m}$ corresponding to $p$ is optimal.

Proof. Suppose there is an operation $y_{1}, \ldots, y_{m}$ of $M$ such that $y_{1}>x_{1}$ and $y_{i} \succsim x_{i}$ for all $i$. Let $p$ be the equilibrium price vector. Then $p \cdot y_{1}<0$, for if $p \cdot y_{1} \geqq 0$ then $y_{1} \in C_{1}(p)$, contradicting the fact that $x_{1}$ is maximal in $C_{1}(p)$. Also $p \cdot y_{i} \leqq 0$ for all $i$, for if $p \cdot y_{i}>0$ then by the hypothesis $y_{i} \notin S_{i}(p)$, hence $x_{i}>y_{i}$ since $x_{i}$ is maximal in $C_{i}(p)$, but this contradicts the assumption on the $y_{i}$. Letting $y=\Sigma y_{i}=\left(\eta_{1}, \ldots, \eta_{n}\right)$ we have $\Sigma_{i} p \cdot y_{i}=p \cdot y<0$ or $\Sigma_{\pi_{j}} \eta_{j}<0$. Since $\pi_{j} \geqq 0$ for all $j$ it follows that $\eta_{j}<0$ for at least one $j$, hence the operation $y_{1}, \ldots, y_{m}$ is not feasible. This proves that the equilibrium operation is optimal.

It is easy to give examples to show that an equilibrium need not be optimal if saving is possible. Thus, consider the case of two individuals $U_{1}$ and $U_{2}$ and a single good $G$. Suppose $U_{1}$ has the whole stock of $G$ and would like to get rid of the amount $\alpha$. Then $S_{1}(p)=S_{1}(1)=\alpha$ while $S_{2}(p)=S_{2}(1)=0$ because of the budget inequality, so the equilibrium bundles are $\alpha$ and 0 . This distribution will not be optimal in case $U_{2}$ desires some positive amount of the good $G$.

\section{BIBLIOGRAPHY}

1. K.J. Arrow and G. Debreu, Existence of an equilibrium for a competitive economy, Econometrica 22 (1954), 265-290.

2. L. M. Graves, The theory of functions of a real variable, New York, 1946.

3. S. Kakutani, A generalization of Brouwer's fixed point theorem, Duke Math. J. 8 (1941), 457-459.

4. B. Knaster, K. Kuratowski und S. Mazurkiewiez, Ein Beweis des Fixpunktsatzes für n-dimensionale Simplexe, Fund. Math. 14 (1931), 132-137.

5. A. Wald, Uber einige Gleichungssysteme der mathematischen Okonomie, Zeitschrift für Nationalökonomie 7 (1936), 637-670. Translation to English, Econometrica 19 (1951), 368-403.

BROWN UNIVERSITY, PROVIDENCE, R.I., U.S.A.

AND

UNIVERSITY OF COPENHAGEN, DENMARK 